\title{
Combination of liquiritin, isoliquiritin and isoliquirigenin induce apoptotic cell death through upregulating p53 and p21 in the A549 non-small cell lung cancer cells
}

\author{
YANLING ZHOU and WING SHING HO
}

School of Life Sciences, The Chinese University of Hong Kong, Shatin, Hong Kong, SAR, P.R. China

Received September 6, 2013; Accepted October 7, 2013

DOI: $10.3892 /$ or.2013.2849

\begin{abstract}
Liquiritin, isoliquiritin and isoliquirigenin are the active polyphenols present in Glycyrrhiza uralensis which has been used for the treatment of cancer and its complications. The present study was conducted to evaluate the cytotoxicity and antitumor activity of liquiritin, isoliquiritin and isoliquirigenin on human non-small lung cancer cells including apoptosis-induction, inhibition of apoptotic pathways and to explore the underlying mechanism. Lactate dehydrogenase assays, FITC Annexin V staining assay were performed to evaluate cellular cytotoxicity and apoptosis activity. The results showed that pretreatment with these polyphenols induced apoptosis in A549 cells. Liquiritin, isoliquiritin and isoliquirigenin significantly increased cytotoxicity of, and upregulated p53 and p21 and downregulated the apoptotic pathways. Furthermore, it inhibited cell cycle at the G2/M phase. Western blot analysis showed it significantly decreased the protein expression of PCNA, MDM2, p-GSK-3 $\beta$, p-Akt, p-c-Raf, p-PTEN, caspase-3, pro-caspase-8, pro-caspase-9 and PARP, Bcl-2 in a concentration-dependent manner while the protein expression of p53, p21 and Bax was increased. In addition, Akt pathway was downregulated. These findings suggest that liquiritin, isoliquiritin and isoliquirigenin inhibited the p53-dependent pathway and showed crosstalk between Akt activities. These active polyphenols can be an alternative agent for the treatment of lung cancer.
\end{abstract}

\section{Introduction}

Lung cancer is one of the leading causes of cancer death. It is the most widely spread carcinoma with a very poor prognosis.

Correspondence to: Professor Wing Shing Ho, School of Life Sciences, The Chinese University of Hong Kong, Shatin, MMW 604, Hong Kong, SAR, P.R. China

E-mail:ws203ho@cuhk.edu.hk

Key words: apoptosis, liquiritin, isoliquiritin, isoliquiritigenin, lung cancer
Although chemotherapy is widely used for treatment of lung cancer, the survival rate is limited (1). Recent study suggested that herbal medicines show promise for lung cancer therapy with less toxicity $(2,3)$. Glycyrrhiza uralensis has been shown to exhibit different pharmacological effects and antioxidant activity against oxidative stress and is used for treatment of various diseases including cancer (4-9). Previous studies showed Glycyrrhiza uralensis contained various types of triterpene, saponins, flavonoids and phenolic compounds (3). However, the major ingredients of Glycyrrhiza uralensis may have different biologic activities. In the present study, we report on experiments designed to test the anticancer activities of the active phenolic compounds of Glycyrrhiza uralensis in the A549 lung cancer cell line.

\section{Materials and methods}

Materials. All chemicals of analytical grade were purchased from USB Corporation (Cleveland, OH, USA). The dried root of Glycyrrhiza uralensis Fisch was obtained from a local pharmaceutical store. Liquiritin, isoliquiritin and isoliquiritigenin were purchased from Shanghai Tauto Biotech Co., Ltd. (Shanghai, China). HPLC grade acetonitrile (Merck, Whitehouse Station, NJ, USA), methanol (Fisher, Fair Lawn, NJ, USA) and trifluoroacetic acid were used for chromatography. Water was purified by Milli-Q Academic purification system (Millipore, Billerica, MA, USA) prior to experiment. Preparation of the active fraction was prepared according to the established method with modifications using different solvent systems (3).

Fetal boving serum (FBS), penicillin streptomycin antibiotic solution (PS), Roswell Park Memorial Institute (RPMI)-1640 media and Trypsin-EDTA were purchased from Invitrogen (Carlsbad, CA, USA). Kaighn's modification of Ham's F-12 Medium (F-12K) was purchased from Gibco Co. (Carlsbad, CA, USA).

Primary antibodies against phospho-AKT (ser473), phospho-AKT (thr308), Akt, p-c-Raf (ser259), p-GSK-3 $\beta$ (ser9), p-Pten (ser380), p-PDK1 (ser241), p53, cleaved caspase-3, caspase-3, PARP, cleaved PARP, caspase-9, cleaved caspase-9, caspase-7, cleaved caspase-7 and anti-rabbit $\lg \mathrm{G}$ were purchased from Cell Signaling Technology, Inc. (Danvers, MA, USA). p21, Bax, Bid and Bcl-2 were purchased from Santa 
Cruz Biotechnology (Santa Cruz, CA, USA). GAPDH was obtained from Ambion/Applied Biosystems (Foster City, CA, USA) and anti-mouse from Zymed Laboratories (Carlsbad, CA, USA).

Cell line and culture. The A549 cell line was obtained from American Type Culture Collection (ATCC, Rockville, MD, USA) and cultured with specific medium with $10 \%$ fetal bovine serum (FBS) and 1\% penicillin-streptomycin (PS) in a $37^{\circ} \mathrm{C}$ incubator with $5 \% \mathrm{CO}_{2}$. Subcultures were performed by using $0.25 \%$ trypsin solution.

MTT assay. The inhibition effects of the ethyl acetate extract were evaluated with the A549 cell line. A549 cells were seeded onto 96-well plate at the concentration of $5 \times 10^{3}$. After $24-\mathrm{h}$ incubation, cells were treated with iquiritin, isoliquiritin and isoliquiritigenin for 24,48 and $72 \mathrm{~h}$, respectively. MTT $(20 \mu \mathrm{l})$ was added to each well and the plate was kept at $37^{\circ} \mathrm{C}$ for $2 \mathrm{~h}$. The purple formazan so formed in the well was dissolved by $150 \mu \mathrm{l}$ of DMSO. The absorbance of the product was measured at $540 \mathrm{~nm}$. Cells without treatment served as vehicle control.

LDH assay. The CytoTox 96 Non-Radioactive cytotoxicity kit (Promega, Madison, WI, USA) was used to evaluate liquiritin, isoliquiritin and isoliquiritigenin cytotoxicity by assessing the total release of cytosolic lactate dehydrogenase in the culture medium. After 24-h incubation, the cell samples were lysed by adding $10 \mu \mathrm{l}$ of Lysis $10 \mathrm{X}$ Solution [9\% (v/v) Triton X-100 in water] per $100 \mu \mathrm{l}$ of culture medium, followed by incubation at $37^{\circ} \mathrm{C}$ for $60 \mathrm{~min}$. The supernatants of the cell sample $(50 \mu \mathrm{l})$ were mixed with the reconstituted substrate $\operatorname{mix}(50 \mu 1)$. The enzymatic assay was stopped after $30 \mathrm{~min}$ by adding $50 \mu \mathrm{l} /$ well of the stop solution (1 M acetic acid). The plate was read at $490 \mathrm{~nm}$ using the plate reader. The amount of LDH release was measured to calculate whole LDH pool in control cells.

Flow cytometric analysis of apoptosis and cell cycle. A549 cells were treated with the active fraction that contained liquiritin, isoliquiritin and isoliquiritigenin for $24 \mathrm{~h}$. Cells were harvested with trypsin and were washed with cold PBS, followed by staining with FITC-PI or PI for the apoptosis or cell cycle study. Fluorescence-activated cells were sorted with 10,000 events per sample in triplicate using a BD Biosciences FACSCanto instrument. The results were analyzed with the FlowJo software version 7.6.1.

Caspase-3/7 assay. The caspase-3/7 substrate Rhodamine 110, Bis-(N-CBZ-L-aspartyl-L-valyl-L-aspartic acid amide; Z-DEVD-R110), is a profluorescent substrate used to study the caspase- 3 activities. Cells were treated with the active mixture prepared according to a previous method (3). After 24-h incubation, Apo-One ${ }^{\circledR}$ Homogeneous Caspase-3/7 assay was performed according to the Promega Techinical protocol. The prepared reagent (100 $\mu \mathrm{l})$ was added to each well (1:1) including the blank, the control or treated cells in culture plate. The mixture was placed in a plate shaker at $300 \mathrm{rpm}$ and incubated at room temperature for $2 \mathrm{~h}$. The fluorescence of each well was measured at excitation $485 \mathrm{~nm}$ and emission $520 \mathrm{~nm}$ using Tecan microplate reader Infinite M200.
Table I. $\mathrm{IC}_{50}$ of Glycyrrhiza uralensis extract and standard compounds in A549 lung cancer cell line.

Fraction

Incubation period $(\mathrm{h})(24 / 48 / 72)$

\begin{tabular}{lc}
\hline EAL $(\mathrm{mg} / \mathrm{ml})$ & 0.049 \\
Lico-1 $(\mathrm{mM})$ & ND \\
Lico-2 $(\mathrm{mM})$ & ND \\
Lico-3 $(\mathrm{mM})$ & $0.093 / 0.065 / 0.037$ \\
Lico-4 $(\mathrm{mM})$ & ND \\
Lico-5 $(\mathrm{mM})$ & $0.14 / 0.087 / 0.074$
\end{tabular}

ND, not detected.

\section{A}

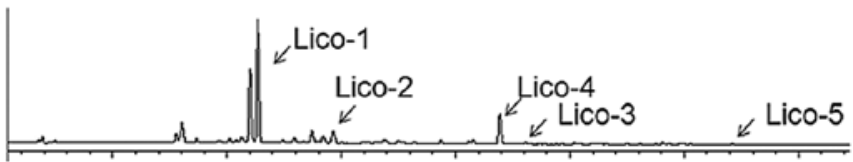

B

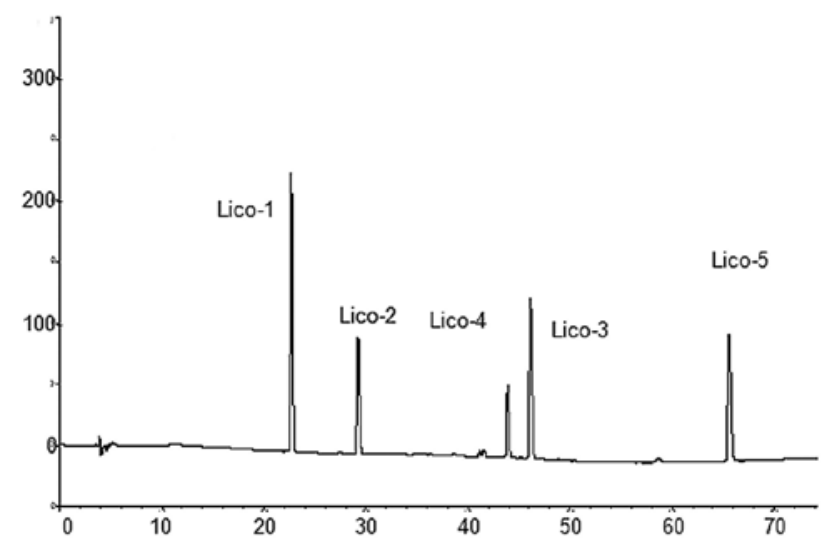

Figure 1. Composition analysis of the ethyl acetate fraction by HPLC with UV detection at $230 \mathrm{~nm}$. (A) liquiritin (lico-1), isoliquiritin (lico-2) and isoliquiritigenin (lico-3) were found. (B) Standard compounds.

Western blot analysis. The cellular proteins were prepared by using lysis buffer [50 mM Trizma base, $100 \mathrm{mM} \mathrm{NaCl}$, $5 \mathrm{mM}$ EDTA, $67 \mathrm{mM}$ sodium pyrophosphate and 1\% Triton $\mathrm{X}-100$, supplemented with protease inhibitor cocktail (Roche, Mannheim, Germany) and $0.5 \mathrm{mM}$ sodium orthovanadate] on ice. The proteins were collected by centrifugation at $8,000 \mathrm{rpm}$ at $4^{\circ} \mathrm{C}$ for $30 \mathrm{~min}$. The protein concentration was determined by the DC Protein assay (Bio-Rad Laboratories) using BSA as the standard. Proteins $(60 \mu \mathrm{g})$ were subjected to $8-15 \%$ SDS-PAGE to separate the protein using 50-120 voltages for $2 \mathrm{~h}$, and were transferred to a PVDF membrane for $45 \mathrm{~min}$ at 12 voltages (Pall Corp., Ann Arbor, MI, USA). After blocking the transfer action with non-fat dry milk (5\% w/v) in TBST for $1 \mathrm{~h}$ at room temperature, the membrane was incubated with 1:1,000 specific primary antibodies with $5 \%$ non-fat milk or BSA in TBST at $4^{\circ} \mathrm{C}$ overnight. After washing with TBST, the membrane was incubated with 1:5,000 HPR-conjugated goat anti-rabbit or goat anti-mouse secondary antibodies for $1 \mathrm{~h}$. Blots were developed using the ECL chemiluminescence 

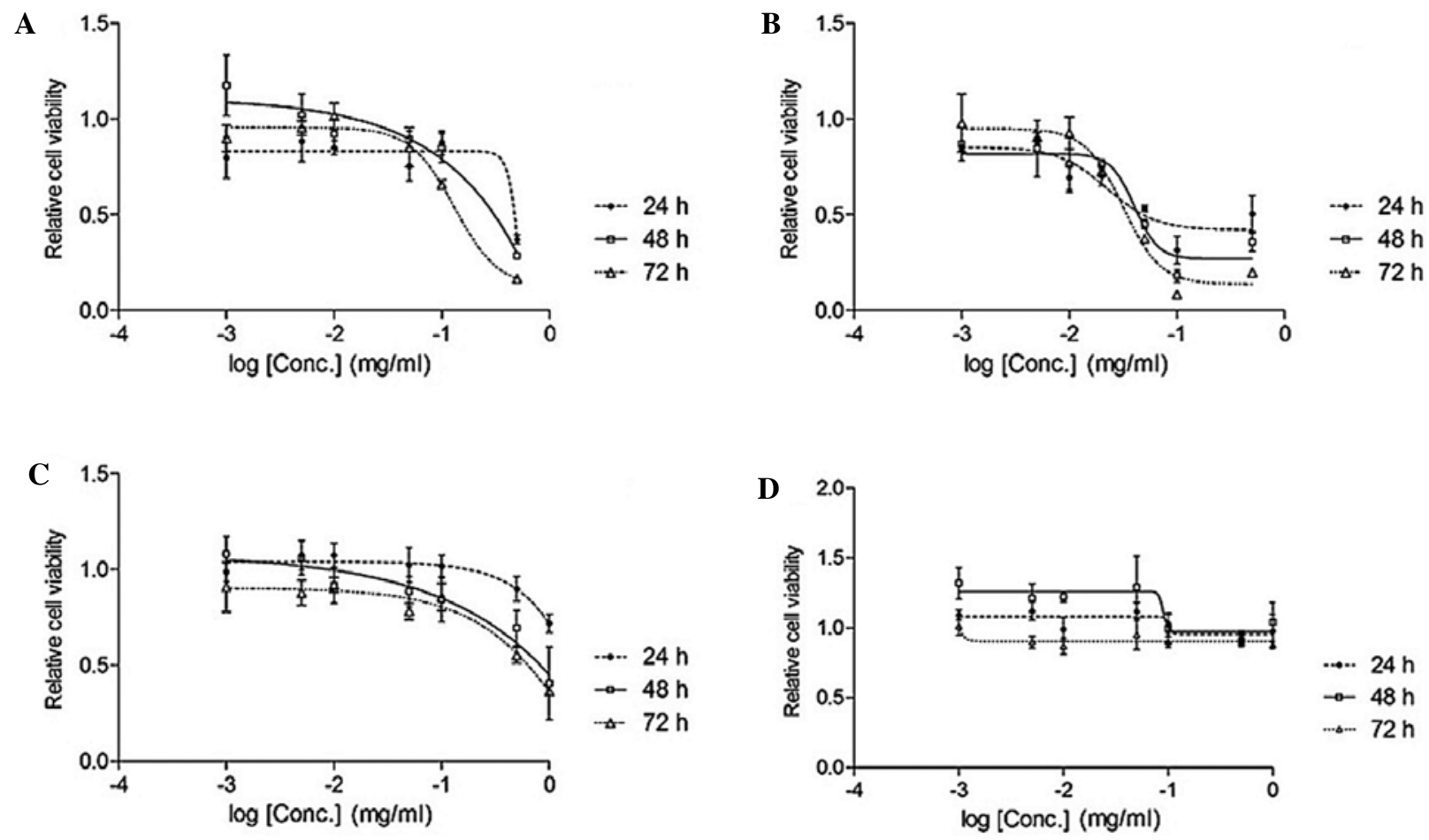

Figure 2. Inhibitory effects of different extracts of Glycyrrhiza uralensis on A549 cell growth. A549 cells were treated with (A) total extract of Glycyrrhiza uralensis (ETL), (B) ethyl acetate fraction (EAL), (C) butanol fraction (BTL) and (D) water fraction of licorice (WAL) or 0.5\% (v/v) DMSO as vehicle control for 24,48 and $72 \mathrm{~h}$. The cell viability was measured using the MTT assay. Data are presented as means $\pm \mathrm{SD}$; $(\mathrm{n}=3)$.

detection reagent (GE Healthcare Life Sciences, Pittsburgh, PA, USA) according to the manufacturer's protocol. The membranes were re-probed with GAPDH as loading reference. The blots were quantified by densitometry and normalized in order to correct any error in protein loading. Densitometric analysis was performed with Gel-Pro 32 software.

Statistical analysis. Statistical analysis of raw data was carried out by one-way analysis of variance (ANOVA) followed by the Tukey's Post Hoc test. The data are expressed as means \pm SD $(n=3)$.

\section{Results}

Cell viability assay. Table I shows the effects of liquiritin, isoliquiritin, isoliquiritigenin and the ethyl acetate extract on the viability of A549 cell lines by MTT assay. Fig. 1 shows that the active fraction contained liquiritin, isoliquiritin and isoliquiritigenin only. The active compounds and the extract inhibited the growth of A549 cells. MTT results show that liquiritin, isoliquiritin and isoliquiritigenin inhibited the lung cancer cell line (Figs. 2 and 3).

LDH leakage assay. The LDH assay shows the cytotoxic effects of the active compounds on A549 cells (Fig. 4). During necrosis, lactate dehydrogenase (LDH) would leak from the inner to the outer of cell since the membrane is broken. Fig. 4 showed that the active extract induced necrosis in A549 cells. Since the LDH leakage was triggered by the noted, a lower concentration range of the active compounds with relatively low LDH leakage, was chosen to evaluate its apoptotic potential.
Annexin V and PI staining. The mode of cell death induced was evaluated to discover how it induced cell death. Annexin V-FITC and propidium iodide staining followed, cytometric analysis were carried out to study the cellular responses. Under the assay condition, the majority of cells were not stained with Annexin V and PI (Fig. 5). During apoptosis, outward translocation of phosphory-serine binds to Annexin V which was conjugated with FITC. In contract, infusion of PI into cells during late apoptosis/necrosis was permitted staining the DNA. The cell viability after $24-\mathrm{h}$ treatment was increased.

Activation of caspase and Bcl-2 family. Bcl-2 family proteins regulate caspase activation. The pro-apoptotic and antiapoptotic protein members in the family determine the fate of the cell. Bcl-2 family includes pro-apoptotic proteins such as Bax, Bak, Bad, Bid and anti-apoptotic proteins such as Bcl-2 and $\mathrm{Bcl}-\mathrm{XL}$. The Bcl-2 family regulates apoptosis through $\mathrm{Bcl}-2$ (inhibitor) or Bax (activator). The ratio of $\mathrm{Bax} / \mathrm{Bcl}-2$ is a key factor in regulation of apoptosis. The expression levels of Bcl-2 family, Bcl-2, Bax and Bid were measured with western blot analysis (Figs. 6-8) and the ratio of Bax/Bcl-2 was recorded. Apo-One Homogeneous Caspase-3/7 assay kit was used to measure caspase-3/7 activities of the A549 cells after treatment. The assay measured the non-fluorescent caspase substrate Z-DEVD-R110 that is cleaved by caspase-3/7, producing the fluorescent Rhodamine 110. Fluorescence generated in the experiment was proportional to the amount of caspase-3/7 cleavage activity in the cultured sample. Fig. 8 shows the increase of fluorescence intensity of caspase- $3 / 7$ after treatment. 

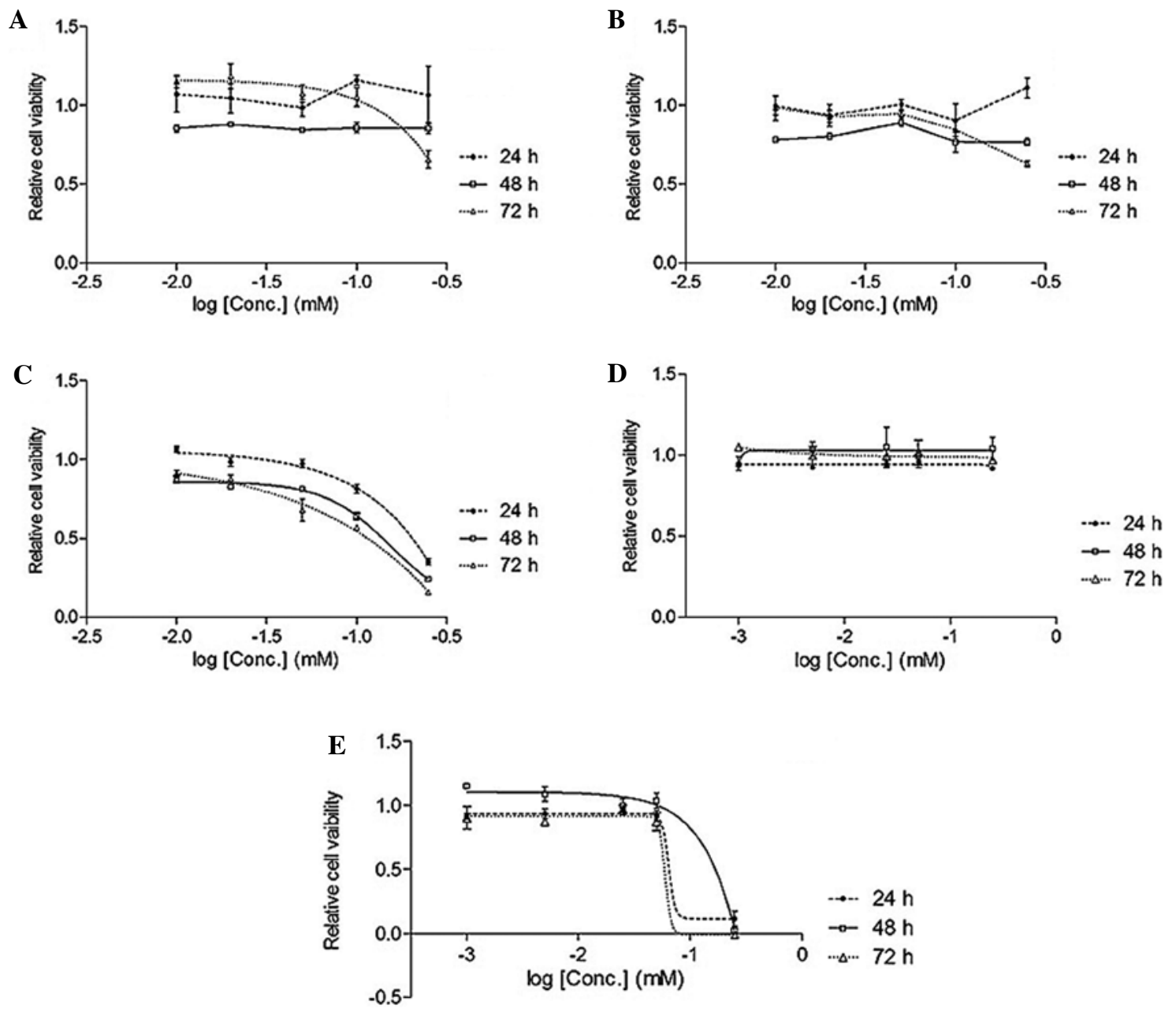

Figure 3. Inhibitory effects of phytochemicals of Glycyrrhiza uralensis on A549 cells growth. A549 cells were treated with (A) lico-1, (B) lico-2, (C) lico-3, (D) lico-4 and (E) lico-5 or 0.5\% (v/v) DMSO as vehicle control for 24,48 and $72 \mathrm{~h}$. The cell viability was measured using the MTT assay. Data are presented as means $\pm \mathrm{SD}(\mathrm{n}=3)$.
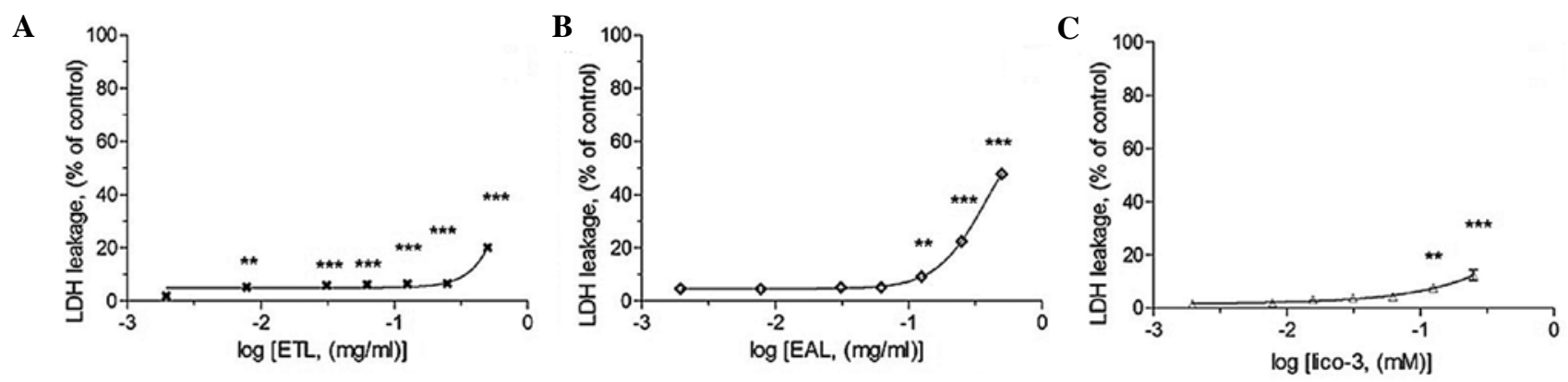

Figure 4. EAL induces cell necrosis of A549 cells. A549 cells were treated with $0.5 \%$ (v/v) DMSO as vehicle control, and (A) ETL, (B) EAL, and (C) lico-3 at $1.95-500 \mu \mathrm{g} / \mathrm{ml}$ for $24 \mathrm{~h}$. The level of LDH release represents the effects of EAL on LDH release, while total release of intracellular LDH caused by $0.9 \%$ (v/v) Triton X-100 from the cells represents the maximal LDH release. The percentage cell death was determined by dividing the experimental LDH release by the maximum LDH release. Data are presented as means $\pm \mathrm{SD}(\mathrm{n}=3) ;{ }^{* * *} \mathrm{P}<0.01$ and ${ }^{* * * *} \mathrm{P}<0.001$.

Caspase family protein expression level in A549 cells after treatment was analyzed by western blotting. The intensity of protein was measured using the Gel-Pro software. The results showed that it caused cleavage of caspase family and PARP.

Suppress Akt survival pathway. The serine/threonine protein kinase Akt (or protein kinase B) activity is related to cancer.
Akt inhibits apoptosis by phosphorylation to initiate multiple additional conversions that directly mediate the action of cell survival or apoptosis. The protein expression level of Akt pathway including Akt phoshorylation forms, p-DK1, GSK-3, PTEN and Raf was studied by western blotting (Fig. 9). EAL could inhibit the phosphorylation of Ak, p-PDK1, p-GSK-3 $\beta$, $\mathrm{p}$-PTEN and p-c-Raf. The ratio of p-Akt (Ser, Thr) over total Akt was measured. 
A
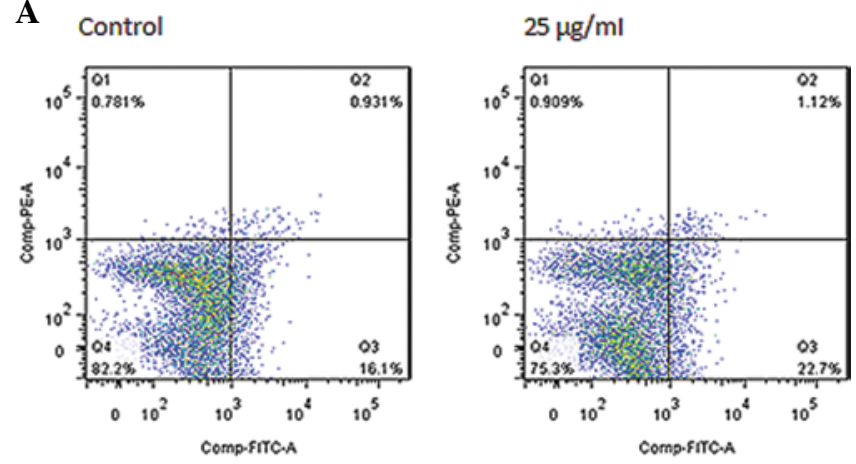

$50 \mu \mathrm{g} / \mathrm{ml}$
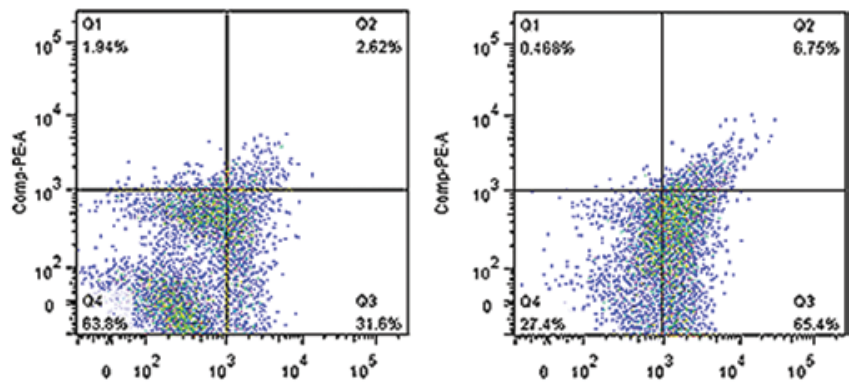

B

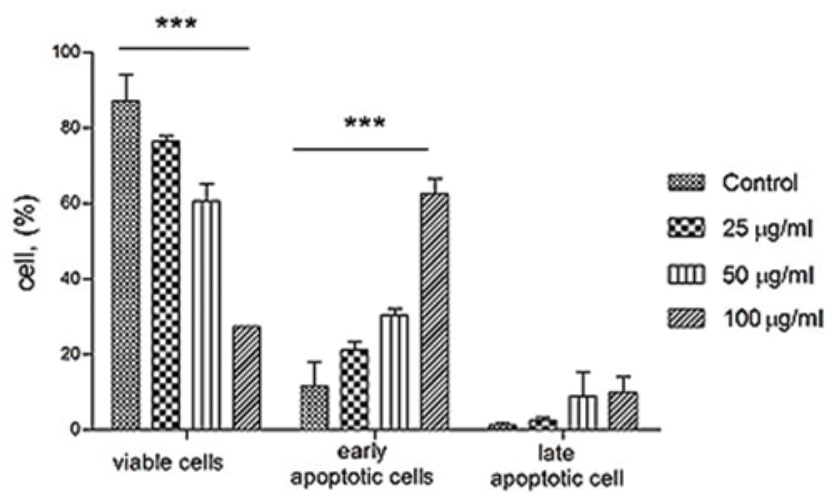

Figure 5. (A) Fluorescence-activated sorting analysis of Annexin V-FITC and propodium iodide (PI) staining for EAL-induced apoptosis of A549. (B) Statistical data analysis of cell populations. A549 cells were treated with 25,50 and $100 \mu \mathrm{g} / \mathrm{ml}$ of EAL or $0.5 \%$ (v/v) DMSO as vehicle control for $24 \mathrm{~h}$. Data are presented as the means $\pm \mathrm{SD}(\mathrm{n}=3) .{ }^{* * *} \mathrm{P}<0.001$.

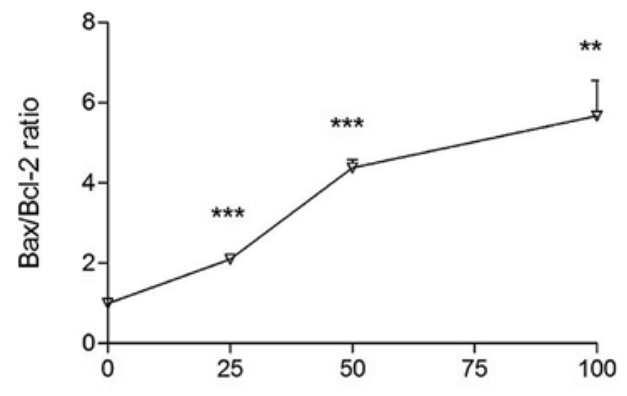

$\mathrm{EAL}(\mu \mathrm{g} / \mathrm{ml})$

Figure 6. Expression of Bax, Bcl-2 after treatment of A549 cells with EAL. A549 cells were treated with different concentrations of EAL or 0.5\% DMSO as vehicle control. Cells were harvested and total proteins were collected. The expression of pro-apoptotic protein Bax and anti-apoptotic protein Bcl-2 and total protein were determined by western blotting. The ratio of Bax to Bcl-2 was determined. ${ }^{*} \mathrm{P}<0.05,{ }^{* *} \mathrm{P}<0.01$ and ${ }^{* * *} \mathrm{P}<0.001$.

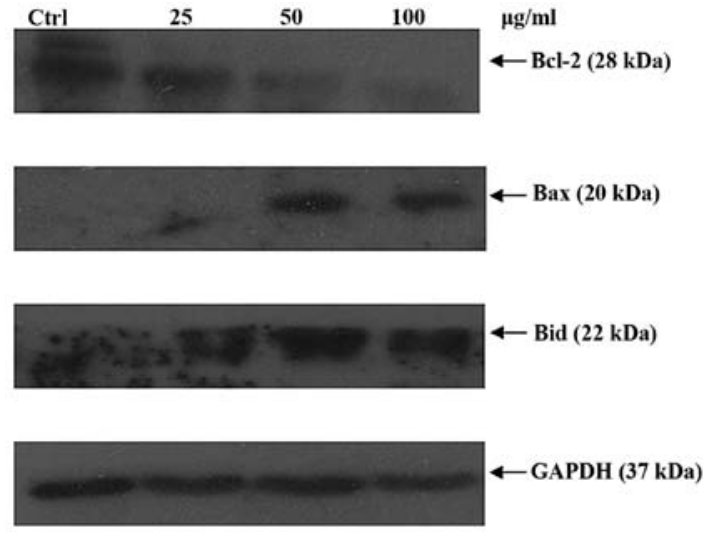

Figure 7. Protein expression of Bcl-2 after treatment of A549 cells with EAL. GAPDH. a housekeeping gene, was used for normalization of protein expression.

A
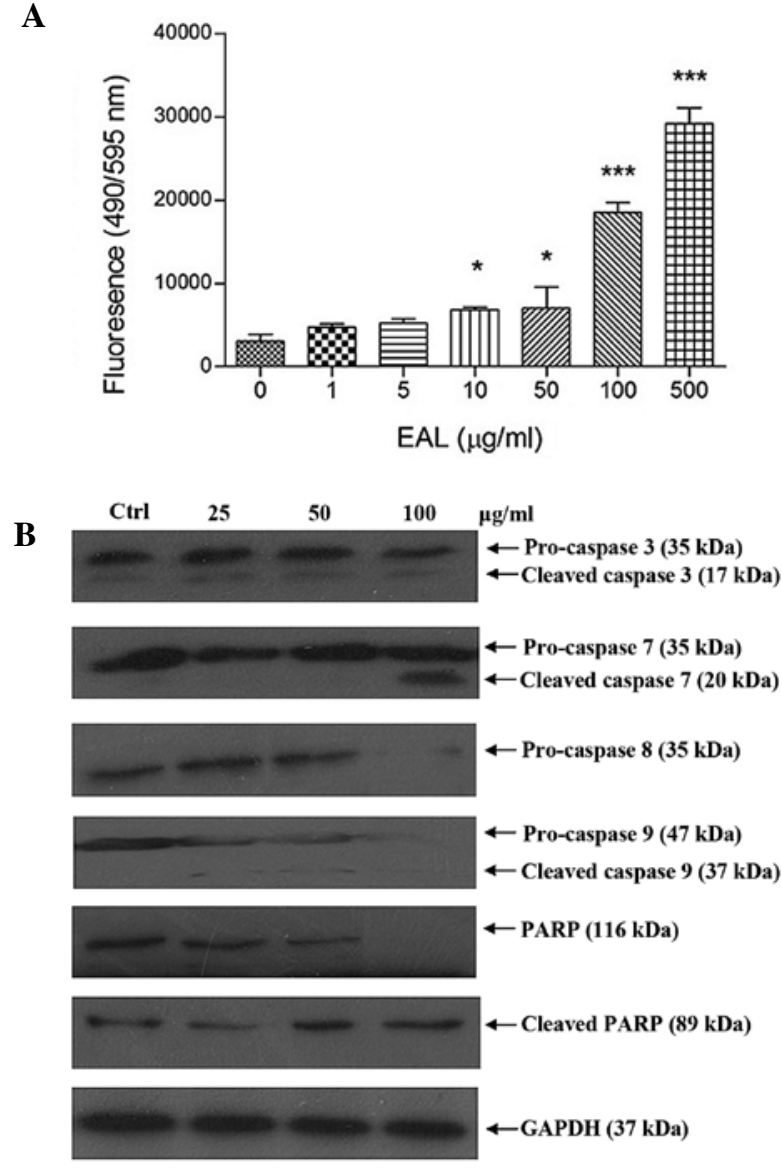

Figure 8. Expression of caspases and PARP after treatment with EAL. (A) The caspase-3/7 activities were measured using the fluorescence microplate reader. (B) Image of western blot analysis. GAPDH was the housekeeping gene used for normalization of protein expression. Data are presented as the means $\pm \mathrm{SD}(\mathrm{n}=3) .{ }^{*} \mathrm{P}<0.05,{ }^{* * *} \mathrm{P}<0.001$.

Cell cycle arrest. To identify whether the growth inhibitory effect is caused by specific perturbation of cell cycle-related events, DNA contents of A549 cells were measured by flow cytometry. p21 is an inhibitor of CDKs which can be regulated by $\mathrm{p} 53$. MDM2, which participates in an auto-regulatory loop with $\mathrm{p} 53$, has been shown to function as a ubiquitin ligase that targets p53 for degradation. The activation and stabilization of 
A

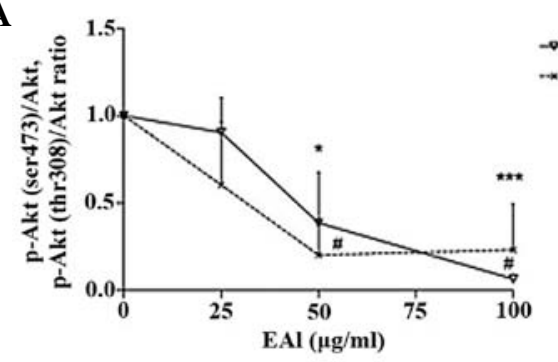

B
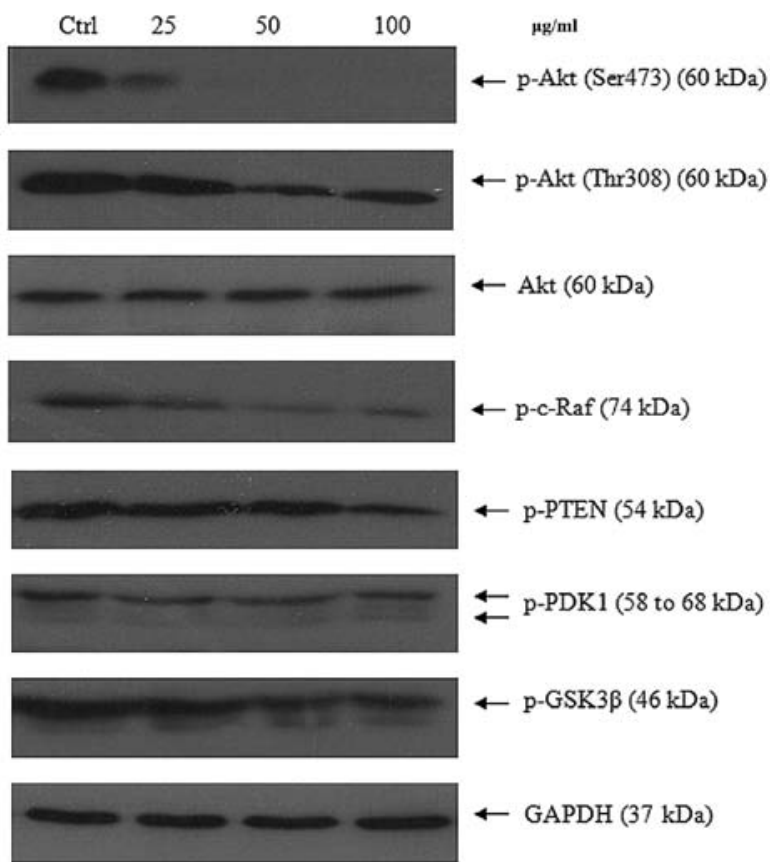

Figure 9. Expression of Akt signaling molecules after treatment of A549 cells with EAL. (A) The ratio of phosphorylation Akt over total Akt was calculated. ${ }^{*}$ $\mathrm{P}<0.05,{ }^{* * *} \mathrm{P}<0.001$. (B) Image of western blot analysis. GAPDH was used for normalization of protein expression.

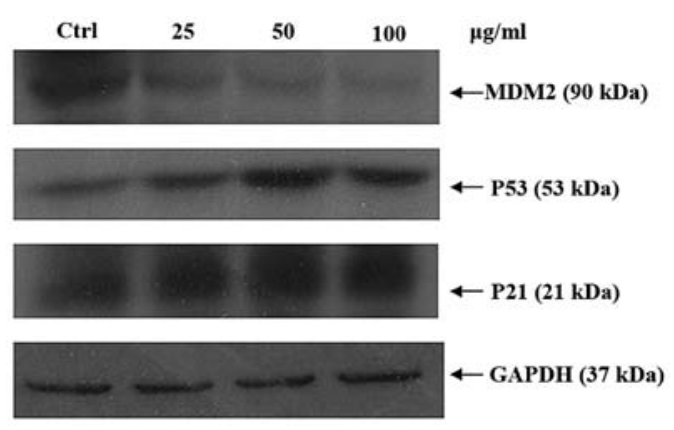

Figure 10. Expression of MDM2, p53 and p21. GAPDH was used for normalization of protein expression.

p53 is believed to play a role in regulating function of MDM2. Any defects in the pathways are believed to influence MDM2 activity in the tumors. MDM2, p53 and p21 protein expression levels were examination by western blot analysis. EAL can upregulate p53 and p21 but downregulated MDM2 (Fig. 10). Proliferation of cell nuclear antigen (PCNA) serves as a processivity factor for DNA polymerase in eukaryotic cells. It played an important role in the cell cycle process, and was a causative factor in PCNA alteration. It reduced PCNA the

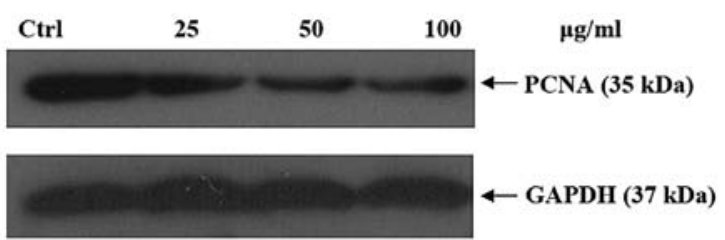

Figure 11. Expression of PCNA. GAPDH was used for normalization of protein expression.

A
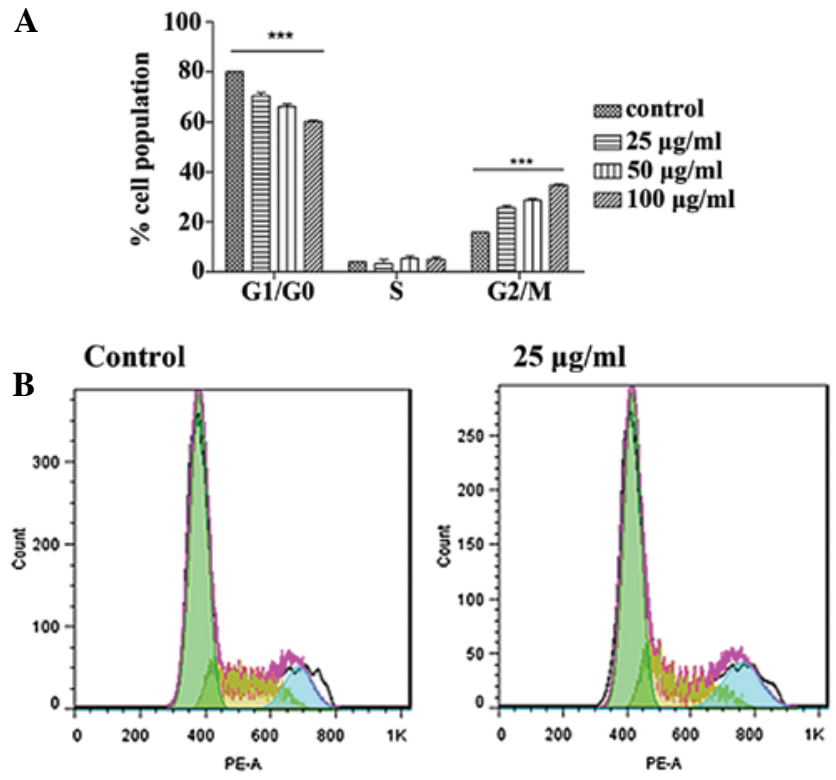

$50 \mu \mathrm{g} / \mathrm{ml}$

$100 \mu \mathrm{g} / \mathrm{ml}$
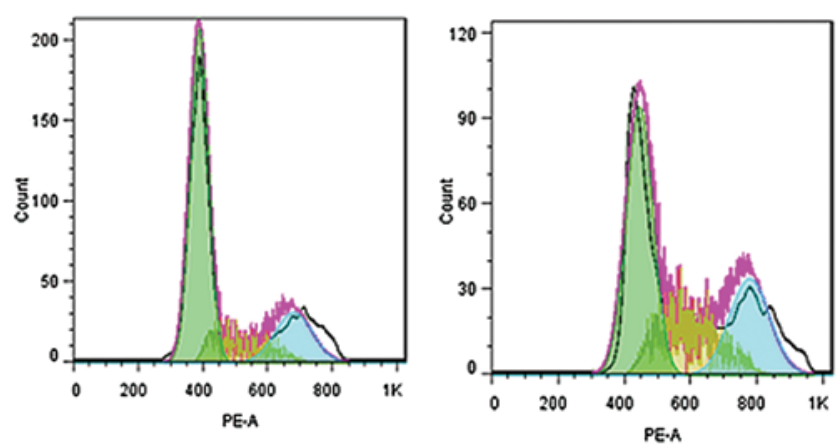

Figure 12. Flow cytometry analysis on Annexin V-FITC and PI staining. Flow cytometry analysis of A549 cells after treatment cells with different concentration of EAL. (A) Statistical analysis of cell population, (B) image of the cell cycle. A549 cells were treated with 25,50 and $100 \mu \mathrm{g} / \mathrm{ml}$ of EAL or $0.5 \%(\mathrm{v} / \mathrm{v}) \mathrm{DMSO}$ as vehicle control for $24 \mathrm{~h}$. Data are presented as the mean $\pm \mathrm{SD}(\mathrm{n}=3) .{ }^{* * *} \mathrm{P}<0.001$.

expression. Fig. 11 shows that it can downregulate the PCNA expression level.

DNA histograms showed that an active fraction containing liquiritin, isoliquiritin and isoliquiritigenin increased the population of $\mathrm{G} 2 / \mathrm{M}$ cells in a concentration-dependent manner at $24 \mathrm{~h}$ (Fig. 12).

\section{Discussion}

The pharmacological activity of active fractions prepared from different methods can vary. Ethyl acetate extraction 
appeared to be more potent and showed relatively higher antitumor activity in non-small cell lung cancer. The composition analysis of the ethyl acetate extract showed it contained three polyphenols, namely, liquiritin, isoliquiritin and isoliquiritigenin. The bioactivities of individual compounds present in Glycyrrhiza uralensis also showed anticancer effects on the lung cancer cell line.

MTT results showed that isoliquiritigenin has the most potent anticancer activity. The present findings suggest that isoliquiritigenin was responsible for the cytotoxicity in the A549 cell line. LDH assay and Annexin V staining confirmed that combination of liquiritin, isoliquiritin and isoliquiritigenin in the active fraction remarkably induced apoptosis.

The expression of related $\mathrm{Bcl}-2$ family anti-apoptotic protein $\mathrm{Bcl}-2$, pro-apoptotic members Bax and Bid, and the cascade activity provide supportive evidence that the Bcl-2 and caspase cascades are involved in apoptosis of the A549 lung cancer cell line. Our results revealed that the active extract containing liquiritin, isoliquiritin and isoliquiritigenin upregulated Bax, Bid protein and downregulated Bcl-2. It increased the enzymatic activity of initiator caspase- 8 and capsase-9. Subsequently it activated the effector caspase-3 and caspase-7, leading to the activation of intrinsic caspase cascades. Caspase- 3 is known to be one of the key executioners of apoptosis because caspase- 3 activation causes the cleavage or degradation of downstream PARP (10). The results suggest the apoptosis of A549 cells is mediated through caspasedependent activity.

The present findings showed that the expression level of Akt pathway is inhibited. A previous study reported that PI3K/Akt signaling cascade is related to cell survival and is mediated via the Bcl-2 family members or caspase family proteins (11). The limitation of full Akt activity is believed to relate to apoptosis of A549 lung cancer cells. Inhibition of Akt can downregulate phosphorylation of GSK-3 $\beta$ at Ser9 (inactive form of GSK-3 $\beta$ ) to trigger apoptosis (12). Akt is known to affect the activity of Erk pathway by silencing c-Raf via phosphorylation at the inhibitory site of Ser259 (13), implicating crosstalk among different signaling pathways. The present findings revealed that deactivation of Akt by Glycyrrhiza uralensis extract may increase the activity of caspase-9 resulting in apoptosis; thus, blocking Akt can increase activities of GSK-3 $\beta$, c-Raf and caspase-9 in A549 cells. Phosphorylated PTEN at Ser380 (suppressive residue) was blocked, suggesting the activation of PTEN through mediation of the Akt pathway. These polyphenols in Glycyrrhiza uralensis caused inhibition of the expression level of phosphorylated PDK1 and Akt resulting in the cell cycle arrest. Altered expression of regulatory cell cycle protein $\mathrm{p} 53$ and $\mathrm{p} 21$ was shown to promote $\mathrm{G} 2 / \mathrm{M}$ phase arrest in A549 cells. Upregulation of p53 and p21 and downregulation of PCNA after treatment suggest it could regulate cell cycle arrest in A549 cancer cells. The findings suggest crosstalk between p53, Bcl-2 family, caspase cascades and the Akt pathway. This could result in apoptosis of cancer cells $(14,15)$. The results suggest that the combination of liquiritin, isoliquiritin and isoliquiritigenin was able to mediate multiple signal pathways resulting in apoptosis.

\section{Acknowledgements}

We are grateful to Luck Tissue Mfy Ltd., for their generosity with a grant no. 6903292 .

\section{References}

1. Broker LE and Giaccone G: The role of new agents in the treatment of non-small cell lung cancer. Eur J Cancer 38: 2347-2361, 2002.

2. Jeong SJ, Koh W, Kim B and Kim SH: Are there new therapeutic options for treating lung cancer based on herbal medicines and their metabolites? J Ethnopharmacol 138: 652-661, 2011.

3. Zhang QY and Ye M: Chemical analysis of the Chinese Herbal medicine Gan-Cao (licorice). J Chromatogr A 1216: 1954-1969, 2009.

4. Yo YT, Shieh GS, Hsu KF, Wu CL and Shiau AL: Licorice and Licochalcone-A induce autophagy in LNCaP prostate cancer cells by suppression of Bcl-2 expression and the mTOR pathway. J Agric Food Chem 57: 8266-8273, 2009.

5. Dong SJ, Inoue A, Zhu Y, Tanji M and Kiyama R: Activation of rapid signally pathways and the subsequent transcriptional regulation for the proliferation of breast cancer MCF-7 cells by the treatment with an extract of Glycyrrhiza glabra root. Food Chem Toxicol 45: 2470-2478, 2007.

6. Fu Y, Hsieh ZC, Guo JQ, Kunicki J, Lee YWT, Darzynkiewicz Z and Wu JM: Licochalcone-A, a novel flavonoid isolated from licorice root (Glycyrrhiza glabra), causes G2 and late-G1 arrests in androgen-independent PC-3 prostat cancer cells. Biochem Biophys Res Commun 322: 263-270, 2004.

7. Hsiang CY, Lai IL, Chao DC and Ho TY: Differential regulation of activator protein 1 activity by glycyrrhizin. Life Sci 70: 1643-1656, 2002

8. Satomi Y, Nishino H and Shibata S: Glycyrrhetinic acid and related compounds induce G1 arrest and apoptosis in human hepatocellular carcinoma HepG2. Anticancer Res 25: 4043-4047, 2005.

9. Yoon G, Bok YK and Seung HC: Topoisomerase I inhibition and cytotoxicity of licochalcones A and E from Glycyrrhiza inflate. Arch Pharm Res 30: 313-316, 2007

10. Chang HY and Yang X: Proteases for cell suicide: functions and regulation of caspases. Microbiol Mol Biol Rev 64: 821-846, 2000.

11. Hsarkar F and Li YW: Targeting multiple signal pathways by chemopreventive agents for cancer prevention and therapy. Acta Pharmacol Sin 28: 1305-1315, 2007.

12. Beurel E and Jope RS: The paradoxical pro- and anti-apoptotic actions of GSK3 in the intrinsic and extrinsic apoptosis signaling pathways. Prog Neurobiol 79: 173-189, 2007.

13. Rommel C, Clarke BA, Zimmermann S, Nuñez L, Rossman R, Reid K, Moelling K, Yancopoulos GD and Glass DJ: Differentiation stage-specific inhibition of the Raf-MEK-ERK pathway by Akt. Science 286: 1738-1741, 1999.

14. Gottlieb TM, Martinez-Leal JF, Seger R, Taya Y and Oren M: Cross-talk between Akt, p53 and MDM2: possible implications for the regulation of apoptosis. Oncogene 21: 1299-1303, 2002.

15. Mayo LD and Donner DB: The PTEN, MDM2, p53 tumor suppressor-oncoprotein network. Trends Biochem Sci 27: 462-467, 2002. 